$(81 \%)$ patients had early stage disease. Fifteen patients $(40.5 \%)$ had a recurrence, with the commonest sites being the pelvis and distant lung metastasis. Seventeen of the patients have died (46\%).

Conclusions In our series there was good correlation between the initial diagnosis and the expert opinion. However, in certain tumour types, specialist review was particularly beneficial in reaching the final diagnosis. This may reflect the enhanced availability of molecular testing at centralised specialist centres. The prognosis is generally unfavourable even in early stage disease.

\section{EPV162/\#343 EDUCATIONAL VALUE OF USING CASE-BASED, RECORDED, OPEN-ACCESS VIDEOMICROSCOPY IN GYNECOLOGIC PATHOLOGY}

L Hassell*. University of Oklahoma Health Sciences Center, Pathology, Oklahoma City, USA Minor Outlying Islands

\subsection{6/ijgc-2021-IGCS.232}

Objectives The COVID-19 pandemic mandated shifting teaching methods to socially distanced modalities. We took the opportunity to create enduring video-microscopy materials of several types using digital slides and offer them via social media to our trainees. Most of the videos also provided links to digital slides for follow-up self-study. After 13 months of providing content, we assess the reach of the effort, and collate responses.

Methods Whole slide images from personal, institutional and public libraries on PathPresenter were used to prepare video presentations, augmented by presentation slides uploaded into the presentation module of the Digital Anatomic Pathology Academy. Video recordings of the presentation were then uploaded to YouTube and the links shared via social media channels (Facebook and Twitter) and email notice to trainees. YouTube channel analytics provided total views, geographic reach of audience and retention times for each video, as well as comments and reactions. Facebook audience reach was also available for videos posted to groups.

Results A total of 89 gynecologic pathology videos were produced and posted, generating a total of 16,718 views, 180 comments, 792 likes and an unknown number of shares. Average audience reach of Facebook-posted videos was 1,500 using a single niche site directed at developing world pathologists. Survey data from group users indicated that most had directly viewed the digital slides.

Conclusions Teaching videos are eagerly received by trainees and practitioners, offer access to unique and common cases, and assist pathology and non-pathology trainees. Patients also gain from the content. Linkage with digital slides is a valued enhancement.

\section{EPV163/\#402 IMPACT OF LYMPH NODE STAGING IN EARLY- STAGE OVARIAN CARCINOMA}

${ }^{1} \mathrm{~F}$ Teixeira*, ${ }^{1} \mathrm{~V}$ De Castro, ${ }^{1} \mathrm{C}$ Faloppa, ${ }^{1} \mathrm{~L}$ Kumagai, ${ }^{1} \mathrm{H}$ Mantoan, ${ }^{1} \mathrm{~L}$ Badiglian-Filho, ${ }^{1} \mathrm{~A}$ Menezes, ${ }^{1} \mathrm{~B}$ Goncalves, ${ }^{2} \mathrm{~A}$ Guimaraes, ${ }^{2} \mathrm{~A}$ Da Costa, ${ }^{1} \mathrm{G}$ Baiocchi. ${ }^{1} \mathrm{AC}$ Camargo Cancer Center, Gynecologic Oncology, Sao Paulo, Brazil; ${ }^{2} A C$ Camargo Cancer Center, Medical Oncology, São Paulo, Brazil

10.1136/ijgc-2021-IGCS.233
Objectives Our aim was to analyze the prevalence of positive lymph node in presumed early-stage ovarian carcinoma (OC) after systematic lymph node dissection (LND) and the impact in adjuvant chemotherapy.

Methods We evaluated a series of 765 patients with OC who underwent surgical treatment from January 2007 to December 2019. Patients with peritoneal disease and incomplete surgical staging were excluded. All cases had systematic pelvic and paraaortic LND up to the renal vessels. After patient referral to our center, a second surgery for staging was done in $37.8 \%$ of cases.

Results A total of 142 cases were ultimately included. The median pelvic and paraaortic lymph nodes (LN) dissected were 30 (range,6-81) and 21 (range,3-86), respectively. Stage shifts after LND and LN metastasis occurred in $8.4 \%$ of cases (12/142) - high-grade serous, $11.9 \%$ (5/42); clear cell, $16.6 \%$ (5/30); endometrioid, 5.1\% (2/39); mixed, 0\% (0/13); and mucinous, $0 \%$ (0/19). Notably, we found clinically suspicious LN (imaging or intraoperative) in 50\% of the metastatic LN. Median hospital stay length was 6 days (range,2-33) and $3.6 \%$ had grade $\geq 3$ complications. Moreover, 110 (77.6\%) patients underwent adjuvant chemotherapy and all cases had indication due to histologic type regardless the result of $\mathrm{LN}$ staging. After a median follow-up of 50.7months (range,1206) we noted 27 (18.9\%) recurrences, and the 5-years recurrence free and overall survival were $92.5 \%$ and $98.1 \%$, respectively.

Conclusions We found a relatively low rate of lymph node positivity and half of positive cases had clinically suspicious LN. The LN status did not impact the indication of adjuvant chemotherapy.

\section{EPV164/\#428 CLINICAL SIGNIFICANCE OF MR IMAGING IN THE JUDGMENT OF LYMPH NODE METASTASIS IN GYNECOLOGICAL MALIGNANT TUMORS}

J Wang*, Y Tang. Chongqing University Cancer Hospital, The Gynecologic Oncology Center Chongqing, China

\subsection{6/ijgc-2021-IGCS.234}

Objectives The lymph node metastasis is closely related to tumor prognosis, and the formulation of postoperative treatment for gynecological malignant tumors. The purpose of this paper is to investigate the clinical value of magnetic resonance imaging in lymph node metastasis of gynecological malignant tumors.

Methods 208 patients undergoing pelvic lymph node and para-aortic lymph node dissection in the Department of Gynecology Chongqing University Cancer Hospital from January 2014 to June 2018 were analyzed retrospectively. SAS9.2 software was used for statistical analysis.

Results The pathological diagnosis in 208 patients showed that 63 patients has pelvic lymph node metastasis, Transfer rate is $30.29 \%$. The sensitivity of MRI to pelvic lymph node transfer is $41.27 \%$.Specificity is $92.41 \%$, Positive forecast value is $70.27 \%$.Negative forecast value is $78.36 \%$.Two-related sample rate test (McNemar test), McNmar Statistics:14.08, $\mathrm{P}=0.0002$, $\mathrm{Kappa}=0.38,95 \% \mathrm{CI}(0.24,0.52)$, The detection rates of the two detection methods differ significantly.37 patients with abdominal aortic lymph node metastasis, Transfer rate is $17.79 \%$.Sensitivity is $29.73 \%$. Specificity is $98.25 \%$.Positive forecast value is $78.57 \%$, Negative forecast value of $86.60 \%$. 